\title{
THE STRENGTH AND STRUCTURE OF THE INTRACLUSTER MAGNETIC FIELD IN THE COMA CLUSTER OF GALAXIES
}

\author{
K.-T. KIM and P. P. KRONBERG \\ Department of Astronomy, \\ University of Toronto \\ Toronto, Ontario M5S 1A7, Canada
}

\begin{abstract}
A new series of detailed observations of the Coma cluster of galaxies has been undertaken with the aim of better specifying the intracluster gas component throgh high dynamic range, combined-telescope observations of the radio halo emission from the cluster which detect both the cluster-scale and galaxy-scale emission. By combining these multi-frequency maps with a Faraday rotation probe experiment using cluster and background sources, and the published X-ray data, we have been able to estimate the intracluster magnetic field strength independently of the unusal assumption of equipartition. The result is approximately 2 microgauss and the tangling of the rms field occurs on an optical galaxy scale.
\end{abstract}

\section{Introduction}

The Coma cluster, due to its relative proximity, is the most accessable "passive laboratory" for studying the intracluster medium (ICM) of a cluster of galaxies. However, even for the Coma cluster the properties of its intracluster magnetic field have been known in less than adequate detail. On the other hand, the existence of an intracluster magnetic field in Coma is evidenced by Coma radio halo which has been observed at various frequencies confirmed the large diameter of this source, its having a steep spectrum, and its being not attributable to the integrated emission of many discrete sources (Willson 1970; Hanisch 1980; Schlickeiser et al. 1987; Kim et al. 1989; and references therein). Hence, the non-thermal Coma halo in which relativistic particles mix with X-ray emitting plasma evidences for the intracluster magnetic field of a substantial strength. The purpose of this paper is to investigate the strength and structure of the intracluster magnetic field, employing the following three different methods: 1 ) direct polarization observations of the radio halo, 2) the equipartition estimate, and 2) Faraday rotation measurements (RMs hereafter) of numbers of sample sources seen in or though the ICM.

\section{Observations and Results}

For the large scale mapping, two instruments, namely the NRAO VLA and the Dominion Radio Astrophysical Observatory (DRAO) interferometer at Penticton, Canada were used to produce a high dynamic range image of the Coma cluster (3000:1) which has good resolution ( $1 \mathrm{arcmin})$ and sensitivity $(30 \mu \mathrm{Jy} / \mathrm{beam})$ to the extended structure (see Fig. 1 and cf. Kim et al. 1989 for detail). The plolarized 


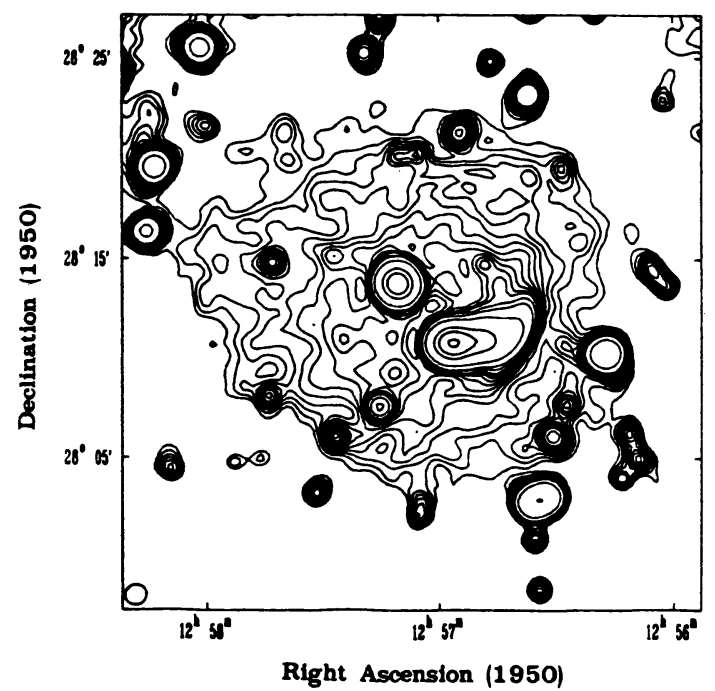

Figure 1. The contour map of Coma halo observed at $1.4 \mathrm{GHz}$. Contours are shown at $-0.6,1.2,1.4,1.6, \ldots$, $3.4,4,5,10,50,100,200 \mathrm{mJy} /$ beam. The restoring beam shown on the lower left corner is $71^{\prime \prime} \times 60^{\prime \prime}$ elongated at $-85.5^{\circ}$ (reproduced from Kim et al. 1989).

component of the halo itself was mapped with the VLA at two frequecies, 1380 and $1665 \mathrm{MHz}$ (method 1 ) but this attempt was unsuccessful due to the absence of any polarized signal from the halo, resulting an upper limit in percentage polarization at $1.4 \mathrm{GHz}$ of $<30 \pm 10 \%$.

Combining all of the existing relevant observations, the halo's spectrum is defined as $\alpha=1.34 \pm 0.06$ down to $1.4 \mathrm{GHz}, F_{1 \mathrm{GHz}}=0.72 \pm 0.13 \mathrm{Jy}$, and the size along the major axis is $\theta_{F W H M}=18^{\prime} .7 \pm 0^{\prime} .1$ at $1.4 \mathrm{GHz}$. These (method 2) yield the best equipartition field strength of $H_{e q}=1.5 \mu G$ for the case of $k=100$, where $k$ is the energy density ratio of relativistic protons to electrons.

In another series of observations with the VLA, we conducted polarimetric mappings at four frequencies $(1465,1665,4835$, and $4885 \mathrm{MHz})$ of 18 discrete sources at varying angular distances from the center of the Coma cluster (method 3 ). These observations yield $17 \mathrm{RMs}$ and a further $18 \mathrm{th}$ was obtained by combining polarization data obtained elsewhere. The excess RM arising from the magneto-ionic medium is estimated to be $\sigma_{c} \approx 38 \pm 6 \mathrm{rad} \mathrm{m}^{-2}$.

Estimate of the turbulence scale was made with the degree of depolarization observed at 4.9 and $1.4 \mathrm{GHz}$ for $5 \mathrm{C} 4.81$, the prominant bent head-tail source. The severity of the observed depolarization (which factor varies from 10 to 20 over the radio-tail) suggets a disordered Faraday screen within which the field reversal was estimated to occur on a physical size range of 13 to $40 \mathrm{kpc}$. Using X-ray core radius obtained with the Einstein observatory (Abramopoulos and $\mathrm{Ku} 1983$ ), this estimate suggets $25 \pm 15$ cells per core radius and when combining with $\sigma_{c}$ the most likely $r m s$ field strength of the intracluster medium turns out to be $H_{c} \approx(1.7 \pm 0.9)$ microgauss. It is noteworthy that this estimate is close to that deduced from the assumption of energy equipartition between the relativistic particles and magnetic field.

\section{References}

Abramopoulos, F., and Ku, W.H.-M. 1983, Ap. J., 271, 446.

Hanisch, R. J. 1980, Astron. J., 85, 1565.

Kim, K., Kronberg, P., Dewdney, P., and Landecker, T. 1989, Ap. J. in press.

Schlickeiser, R., Sievers, A., and Thiemann, H. 1987, Astron. Astrophys. 182, 21. Willson, M.A.G. 1970, Mon. Not. R. Astron. Soc., 151, 1. 
BURNS: There is an interesting additional observational constraint on occurance of cluster-wide halos. That is, of the half-dozen known halos, none are in cooling flows. Such flows seem to surpress large-scale halos. Perseus is a good example of a cluster with dozens of radio galaxies (extensive seeds for halos) yet it has no halos. This anticorrelation is hard to understand since the cooling region of clusters is usually $<200 \mathrm{kpc}$, much less than the extent of halos like that in Coma.

SHUKUROV: One of the objections against the galactic-wake theory of intracluster turbulence is the fact that there are no "tail" radio sources without "heads". However, in central parts of galaxy clusters the wakes can merge to produce a quasi-uniform turbulent picture and only in the outer parts, where the number density of galaxies is smaller, the wakes can possibly preserve their individuality. However, the gas density is lower at the outskirts and it can be difficult to observe these individual wakes. Does this speculation fit the observed picture?

KIM: If the hypothetical radio tails exist, then we should see them, unless the radio tails are developing at a distance far away from the moving galaxy. The answer might be like this: In order for the induced turbulence to be fully developed it requires time scales of the order of $10^{8}$ yrs during which a parent galaxy can move $\sim 100 \mathrm{kpc}$. So if this idea is correct then we are likely to see radio tails well detached from the galaxy.

KRONBERG: Our data show clear evidence for discrete intervening systems out to $\mathrm{z} \sim 3$, but no evidence so far for a general intergalactic field out to large redshifts. 